\title{
Cutter-Torque Cable Device
}

National Cancer Institute

\section{Source}

National Cancer Institute. Cutter-Torque Cable Device. NCI Thesaurus. Code C50342.

A cable designed to drive the rotational cutter of a catheter device. 